NISTIR 8259A

\title{
Linha de Base do Núcleo de Recursos de Cibersegurança de Dispositivos IoT
}

\author{
Michael Fagan \\ Katerina N. Megas \\ Karen Scarfone \\ Matthew Smith
}

Esta publicação está disponível gratuitamente em: https://doi.org/10.6028/NIST.IR.8259Apt 
NISTIR 8259A

\title{
Linha de Base do Núcleo para Recursos de Cibersegurança de Dispositivos IoT
}

\author{
Michael Fagan \\ Katerina N. Megas \\ Divisão de Cibersegurança Aplicada \\ Laboratório de Tecnologia da Informação \\ Karen Scarfone \\ Scarfone Cybersecurity \\ Clifton, VA \\ Matthew Smith \\ Huntington Ingalls Industries \\ Annapolis Junction, MD
}

Esta publicação está disponível gratuitamente em:

https://doi.org/10.6028/NIST.IR.8259Apt

Maio 2020

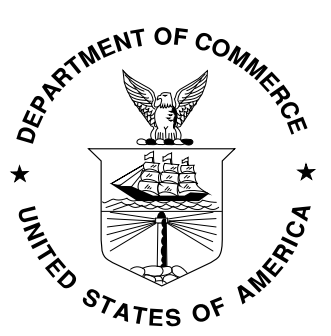

Departamento de Comércio dos EUA Wilbur L. Ross, Jr., Secretário

Instituto Nacional de Normas e Tecnologia Walter Copan, Diretor do NIST e Subsecretário de Comércio para Normas e Tecnologia 


\title{
Relatório Interno ou Interagências 8259A do Instituto Nacional de Normas e Tecnologia 23 páginas (Maio 2020)
}

\section{Esta publicação está disponível gratuitamente em: https://doi.org/10.6028/NIST.IR.8259Apt}

Certas entidades comerciais, equipamentos ou materiais podem ser identificados neste documento para descrever adequadamente determinado procedimento ou conceito experimental. Esta identificação não tem a intenção de sugerir recomendação ou endosso do NIST, nem tem a intenção de sugerir que as entidades, materiais ou equipamentos sejam necessariamente os melhores disponíveis para tal propósito.

Esta publicação pode conter referências a outras publicações atualmente sendo produzidas pelo NIST de acordo com as responsabilidades estatutárias que lhe foram atribuídas. As informações nesta publicação, incluindo conceitos e metodologias, podem ser usadas por agências federais mesmo antes da conclusão de tais publicações complementares. Assim sendo, até que cada publicação seja concluída, os requisitos, diretrizes e procedimentos atuais, onde existam, permanecem operacionais. Para fins de planejamento e transição, as agências federais podem desejar acompanhar de perto o desenvolvimento das novas publicações produzidas pelo NIST.

As organizações são incentivadas a revisar todos os esboços preliminares das publicações durante os períodos de comentários públicos e fornecer feedback ao NIST. Muitas publicações do NIST sobre segurança cibernética, além das mencionadas acima, estão disponíveis em https://csrc.nist.gov/publications.

\section{Os comentários sobre esta publicação podem ser enviados para:}

\author{
Instituto Nacional de Normas e Tecnologia \\ A/C: Divisão de Segurança Cibernética Aplicada, Laboratório de Tecnologia da Informação \\ 100 Bureau Drive (Mail Stop 2000) Gaithersburg, MD 20899-2000 \\ E-mail: iotsecurity@nist.gov \\ Todos os comentários estão sujeitos a divulgação no âmbito da Lei de Liberdade de \\ Informação (FOIA).
}

\section{Disclaimer}

This document was translated by the U.S. Department of State, Office of Language Services with support from the Digital Connectivity and Cybersecurity Partnership (DCCP).

The official English language version of this publication is available free of charge from the National Institute of Standards and Technology (NIST): https://doi.org/10.6028/NIST.IR.8259A. 


\section{Relatórios sobre Tecnologia de Sistemas Informáticos}

O Laboratório de Tecnologia da Informação (ITL) do Instituto Nacional de Normas e Tecnologia (NIST) promove a economia e o bem-estar público dos EUA, fornecendo liderança técnica para a infraestrutura de medição e normas da Nação. O ITL desenvolve testes, métodos de testes, dados de referência, implementações de prova de conceito e análises técnicas para promover o desenvolvimento e o uso produtivo da tecnologia da informação. As responsabilidades do ITL incluem o desenvolvimento de padrões e diretrizes de gestão, administrativos, técnicos e físicos para a segurança economicamente viável e a privacidade de outras informações, além das relacionadas à segurança nacional em sistemas federais de informação.

\section{Resumo}

Os recursos de cibersegurança dos dispositivos são funções que os dispositivos de computação fornecem através dos seus próprios meios técnicos (ex: hardware e software do dispositivo). Esta publicação define uma linha de base dos recursos de cibersegurança do dispositivo da Internet das Coisas (IoT), que é um conjunto de recursos geralmente necessários para oferecer suporte a controles comuns de segurança cibernética que protegem os dispositivos de uma organização, bem como dados, sistemas e ecossistemas de dispositivos. O objetivo desta publicação é fornecer às organizações um ponto de partida na identificação dos recursos de segurança cibernética para novos dispositivos IoT que irão fabricar, integrando ou adquirindo. Esta publicação pode ser usada juntamente com o NISTIR 8259, Foundational Cybersecurity Activities for IoT Device Manufacturers [Atividades básicas de segurança cibernética para fabricantes de dispositivos IoT].

\section{Palavras-chave}

Cibersegurança, Internet das Coisas (IoT); dispositivos de computação protegíveis.

\section{Reconhecimentos}

Os autores desejam agradecer a todos os colaboradores desta publicação, incluindo os participantes em workshops e outras sessões interativas, os indivíduos e organizações dos setores público e privado, incluindo fabricantes de vários setores da indústria, além de diversas organizações comerciais de fabricantes que forneceram feedback sobre o ensaio preliminar e os esboços de comentários públicos. Agradecemos também aos colegas do NIST que nos proporcionaram informações e feedback importantes. Queremos agradecer, especialmente, aos integrantes da equipe do Programa de Cibersegurança para IoT, Barbara Cuthill e Jeff Marron. e à equipe do Projeto de Implementação do NIST FISMA por sua ajuda na editoração deste trabalho.

\section{Público-alvo}

Os fabricantes de dispositivos IoT são o público-alvo desta publicação, O conteúdo desta publicação também pode ajudar os clientes ou integradores de dispositivos IoT. 
Tabela de Conteúdo

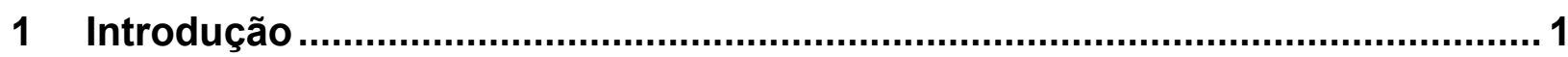

2 Definição da linha de base do núcleo para recursos de cibersegurança dos

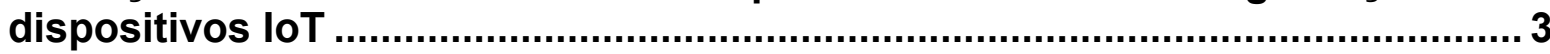

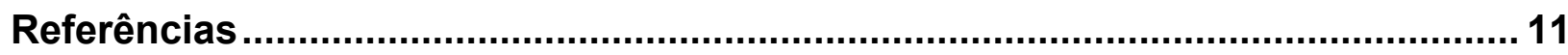

Appendix A-Compreendendo a linha de base do núcleo para recursos de cibersegurança de dispositivo loT no contexto das necessidades e objetivos

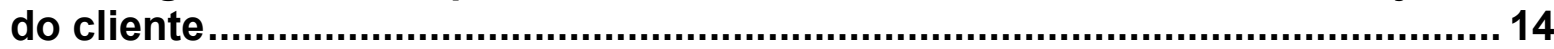

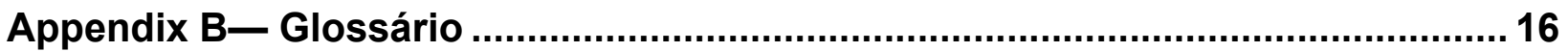




\section{Aviso de divulgação de patente}

AVISO: A ITL solicitou que os detentores de reivindicações de patentes, cujo uso possa ser necessário para o cumprimento das diretrizes ou requisitos desta publicação, divulguem tais reivindicações de patente à ITL. No entanto, os detentores de patentes não são obrigados a responder aos pedidos da ITL referentes a patentes, e a ITL não realizou uma pesquisa de patentes para identificar quais são as patentes (se houver) que se aplicam a esta publicação.

Até a data desta publicação e das subsequentes solicitações para identificação de reivindicações de patentes cujo uso possa ser necessário para o cumprimento das diretrizes ou requisitos desta publicação, nenhuma reivindicação de patente foi identificada como sendo da ITL.

Nenhuma declaração foi feita pela ITL ou está implícita de que as licenças não são necessárias para evitar violação de patente no uso desta publicação. 


\section{Introdução}

Os dispositivos de computação que integram recursos físicos e/ou de detecção e recursos de interface de rede estão sendo projetados, desenvolvidos e implantados em um ritmo cada vez maior, para atender às necessidades dos clientes em todos os setores da economia, sendo que muitos dispositivos de computação estão conectados à Internet. Uma nova característica desses dispositivos é a combinação de conectividade e a capacidade de perceber e/ou afetar o mundo físico. Conforme os dispositivos se tornam menores e mais complexos, e devido ao número crescente de recursos, a segurança para os dispositivos também se torna mais complexa. Esta publicação define um conjunto básico de recursos de cibersegurança de dispositivos que as organizações devem considerar ao enfrentarem o desafio da Internet das Coisas (IoT).

Os recursos de cibersegurança de dispositivo são funções de cibersegurança que os dispositivos de computação fornecem através dos seus próprios meios técnicos (ex: hardware e software do dispositivo). A linha de base do núcleo para recursos de cibersegurança dos dispositivos IoT (linha de base do núcleo) ${ }^{1}$ definida nesta publicação, consiste em um conjunto de recursos do dispositivo geralmente necessários para dar suporte aos controles de segurança cibernética comumente usados que protegem os dispositivos, bem como os dados, sistemas e ecossistemas dos dispositivos. O conceito de linha de base em qualquer contexto requer consideração meticulosa, e os recursos de segurança para dispositivos IoT não são exceção.

A linha de base do núcleo foi derivada de pesquisas sobre abordagens comuns de gerenciamento de risco de cibersegurança e recursos comumente usados para abordar os riscos de cibersegurança para dispositivos IoT, que foram refinados e validados usando um processo público-privado colaborativo para incorporar todos os pareceres. Recebemos vários pedidos para comentários e realizamos diversos workshops e mesa-redonda. O NIST assume o compromisso de realizar um processo aberto, transparente e interativo que facilita o feedback das partes interessadas.

Esses recursos foram desenvolvidos no contexto do NISTIR 8259, Foundational Cybersecurity Activities for IoT Device Manufacturers [Atividades básicas de segurança cibernética para fabricantes de dispositivos IoT] [2], que discute possibilidades para os fabricantes, orientando-os na escolha e implementação dos recursos de cibersegurança fornecidos pelos seus dispositivos IoT. O NISTIR 8259 também define a terminologia e os conceitos em um contexto crítico para melhor compreensão dos recursos de cibersegurança do dispositivo como uma parte de todo o ecossistema de segurança cibernética da IoT. Portanto, embora o NISTIR 8259 e esta publicação tenham como público-alvo os fabricantes de dispositivos, as considerações e recursos discutidos nas duas publicações podem ser usados por fabricantes, integradores e consumidores. Para mais informações sobre como esses recursos podem ser incorporados aos processos de

O uso do termo "linha de base" nesta publicação não deve ser confundido com as linhas de base de controles de sistemas de baixo, moderado e alto impacto descritas na Publicação Especial NIST (SP) 800-53 [1] para ajudar os órgãos federais a cumprir suas obrigações no âmbito da Lei Federal de Modernização da Segurança da Informação (FISMA) e outras legislações federais. Nesse contexto, as linhas de base de controle de baixo, moderado e alto impacto se aplicam a um sistema de informação que pode incluir vários componentes, inclusive os dispositivos. Nesta publicação, a "linha de base" é usada no sentido genérico para se referir a um conjunto de requisitos ou recomendações fundamentais, sendo que a linha de base do núcleo aqui descrita deve ser aplicada aos dispositivos IoT individuais. 
desenvolvimento de um fabricante, consulte o NISTIR 8259. Outras organizações podem usar a linha de base do núcleo no contexto em que ela está disponível e que for mais adequado.

Independentemente da função de uma organização, esta linha de base tem como objetivo proporcionar a todas as organizações um ponto de partida para o gerenciamento de risco cibersegurança dos dispositivos IoT, porém, a implementação de todos os recursos não é considerada obrigatória. Os recursos individuais da linha de base podem ser implementados total ou parcialmente, ou não implementados. Fica a critério da organização implementadora entender o contexto peculiar de risco no qual ela opera e o que é adequado para a sua circunstância. Para obter mais informações sobre como realizar uma avaliação de risco, consulte a Publicação Especial NIST 800-30, Guide for Conducting Risk Assessments [Guia para realizar avaliações de risco] [3].

Além disso, essa linha de base não é o único conjunto de recursos disponíveis. Ela representa um esforço coordenado para chegarmos a uma definição de quais são os recursos comuns, porém, esta não é uma lista exaustiva. Portanto, uma organização que faz a implementação pode ser mais indicada para definir os recursos que melhor se adaptam à sua organização.

Incentivamos o uso desses recursos adicionais para oferecer suporte ao gerenciamento de risco de cibersegurança dos dispositivos IoT. Para obter mais informações sobre segurança dos dispositivos IoT e considerações de privacidade, consulte o NISTIR 8228, Considerations for Managing Internet of Things (IoT) Cybersecurity and Privacy Risks [Considerações para Gerenciar Riscos de Privacidade e Segurança Cibernética na Internet das Coisas (IoT)] [4]. 


\section{Definição da linha de base do núcleo para recursos de cibersegurança dos dispositivos loT}

Table 1 Define a linha de base do núcleo para recursos de cibersegurança dos dispositivos IoT. A linha de base do núcleo é o padrão para dispositivos minimamente seguros. No entanto, os recursos de cibersegurança dos dispositivos muitas vezes precisam ser acrescentados ou retirados do design, integração ou aquisição de um dispositivo IoT para melhor solucionar os riscos comuns de segurança cibernética de uma organização. A linha de base do núcleo não especifica como os recursos de segurança cibernética dos dispositivos devem ser alcançados, portanto, as organizações que optam por adotar a linha de base do núcleo para qualquer um dos dispositivos IoT que produzem, integram ou adquirem, têm flexibilidade considerável para implementá-la e efetivamente atender às suas necessidades.

Cada fileira da Table 1 descreve um dos recursos de cibersegurança do dispositivo na linha de base do núcleo:

- A primeira coluna define o recurso. Observe que a Figure 1, localizada no Apêndice A, indica como o recurso se relaciona às áreas de mitigação de risco e desafios definidos no NISTIR 8228, Considerations for Managing Internet of Things (IoT) Cybersecurity and Privacy Risks [Considerações para Gerenciar Riscos de Privacidade e Segurança Cibernética na Internet das Coisas (IoT)] [3].

- A segunda coluna mostra uma lista enumerada de elementos comuns relativos a tais recursos - elementos que uma organização que esteja planejando implementar a linha de base do núcleo várias vezes (mas nem sempre) usaria para conseguir os recursos desejados. (Nota: os elementos não são abrangentes, nem estão em uma ordem específica.)

- A terceira coluna explica o raciocínio para a necessidade dos recursos e seus elementos comuns a serem incluídos na linha de base do núcleo.

- A última coluna lista exemplos de referências e fontes para obter orientação sobre cibersegurança para dispositivos IoT, especificando recursos semelhantes ou relacionados. Como a tabela aborda apenas os elementos básicos dos recursos, as referências são importantes para haver melhor compreensão e detalhes sobre cada recurso, e para aprender como implementálos da maneira adequada. Essas são as referências usada na Table 1:

○ AGELIGHT: AgeLight Digital Trust Advisory Group, "IoT Safety Architecture \& Risk Toolkit (IoTSA)" [Arquitetura de segurança de IoT e kit de ferramentas de risco] v3.1" [5]

○ BITAG: Broadband Internet Technical Advisory Group (BITAG), "Internet of Things (IoT) Security and Privacy Recommendations" [Recomendações de segurança e privacidade da Internet das coisas (IoT)] [6]

- CSA: Cloud Security Alliance (CSA) Grupo de trabalho de IoT, "Identity and Access Management for the Internet of Things" [Gerenciamento de identidade e accesso para an Internet das Coisas] [7] 
- CSDE: Council to Secure the Digital Economy (CSDE), "The C2 Consensus on IoT Device Security Baseline Capabilities" (Consenso do C2 sobre Recursos de linha de base de segurança do dispositivo IoT [8]

○ CTIA: CTIA, “CTIA Cybersecurity Certification Test Plan for IoT Devices, Version 1.0.1 (Teste de certificação de cibersegurança para dispositivos IoT) " [9]

- ENISA: Agência da União Europeia para a Segurança das Redes e da Informação (ENISA), "Baseline Security Recommendations for IoT in the context of Critical Information Infrastructures" [Recomendações de Segurança da linha de base para IoT no contexto de Infraestruturas Críticas de Informação] [10]

- ETSI: Instituto Europeu de Normas de Telecomunicações (ETSI), "Cyber Security for Consumer Internet of Things" [Segurança cibernética para o consumidor da Internet das Coisas] [11]

○ GSMA: Groupe Spéciale Mobile Association (GSMA), "GSMA IoT Security Assessment” [Avaliação de segurança IoT da GSMA] [12]

○ IEC: Comissão Eletrotécnica Internacional (IEC), "IEC 62443-4-2, Edition 1.0, Security for industrial automation and control systems - Part 4-2: [IEC 62443-4-2, Edição 1.0, Segurança para automação industrial e sistemas de controle - Parte 4-2:] [Technical security requirements for IACS components] [13]

○ IIC: Consórcio da Internet Industrial (IIC), “Internet das Coisas Industrial Volume G4: Security Framework” [14]

○ IoTSF: Base de Segurança IoT (IoTSF), “Estrutura de Compliance em Segurança de IoT, Lançamento 2” [15]

○ ISOC/OTA: Sociedade da Internet /Aliança de Confiança Online (OTA), "Estrutura de Privacidade e Segurança de IoT $\mathrm{v} 2.5 "[16]$

○ NEMA: Associação Nacional de Fabricantes Elétricos (NEMA), "Melhores Práticas de Higiene Cibernética" [17]

○ OCF: Fundação de Conectividade Aberta (OCF) "Especificações de Segurança da OCF Versão 2.1.2” [18]

- PSA: Platform Security Architecture (PSA) Arquitetura de Segurança de Plataforma Membros do Acordo Conjunto das Partes Interessadas (JSA), “Certificação PSA TM Questionário de Nível I Versão 2.0 Beta” [19]

O Apêndice B contém definições para os itens sublinhados na Table 1. 
Tabela 1: A linha de base do núcleo para recursos de cibersegurança dos dispositivos loT seguros

\begin{tabular}{|c|c|c|c|}
\hline $\begin{array}{l}\text { Recurso de } \\
\text { cibersegurança do } \\
\text { dispositivo }\end{array}$ & Elementos comuns & Raciocínio & Exemplos de referência loT \\
\hline $\begin{array}{l}\text { Identificação do } \\
\text { dispositivo: O } \\
\text { dispositivo loT pode } \\
\text { ser identificado de } \\
\text { forma exclusiva, de } \\
\text { maneira lógica e física. }\end{array}$ & $\begin{array}{l}\text { 1. Um identificador lógico exclusivo } \\
\text { 2. Um identificador físico exclusivo em } \\
\text { um local externo ou interno no } \\
\text { dispositivo que as entidades } \\
\text { autorizadas podem acessar } \\
\text { Observação: os identificadores físicos e } \\
\text { lógicos podem representar o mesmo } \\
\text { valor, mas não necessariamente. }\end{array}$ & $\begin{array}{l}\text { - Esse recurso oferece suporte ao gerenciamento de } \\
\text { ativos, que por sua vez oferece suporte ao } \\
\text { gerenciamento de vulnerabilidades, de acesso, } \\
\text { proteção de dados e detecção de incidentes. } \\
\text { - O identificador lógico exclusivo pode ser usado para } \\
\text { distinguir o dispositivo de todos os outros, } \\
\text { geralmente para gerenciamento e monitoramento } \\
\text { automatizado de dispositivo. Isso pode exigir que } \\
\text { seja imutável para permitir uma identificação } \\
\text { consistente usando o identificador. O identificador } \\
\text { lógico exclusivo também pode ser usado para } \\
\text { autenticação do dispositivo, mas deve-se considerar } \\
\text { a seleção de um identificador apropriado para tal } \\
\text { propósito. } \\
\text { - O identificador físico exclusivo pode ser usado para } \\
\text { distinguir o dispositivo de todos os outros sempre } \\
\text { que o identificador lógico exclusivo não estiver } \\
\text { disponível, como durante a implantação e } \\
\text { desativação do dispositivo ou após uma falha. } \\
\text { - A capacidade também pode precisar de um } \\
\text { identificador lógico adicional que não será } \\
\text { necessariamente exclusivo, para ser usado para } \\
\text { propósitos mais específicos, como sinalização de } \\
\text { intenção do dispositivo. }\end{array}$ & $\begin{array}{l}\text { - CSA: } 1 \\
\text { - CSDE: } 5.1 .1 \\
\text { - CTIA: } 4.13 \\
\text { - ENISA: GP-PS-10 } \\
\text { - GSMA: CLP13_6.6.2, 6.8.1, } \\
\text { 6.20.1 } \\
\text { - IEC: CR } 1.2 \\
\text { - IIC: } 7.3,8.5,11.7,11.8 \\
\text { - IoTSF: } 2.4 .8 .1,2.4 .14 .3 \\
\text { 2.4.14.4 } \\
\text { - OCF: } 7.1 .1 \\
\text { - PSA: C1.4, R2.1 }\end{array}$ \\
\hline
\end{tabular}




\begin{tabular}{|c|c|c|c|}
\hline $\begin{array}{l}\text { Recurso de } \\
\text { cibersegurança do } \\
\text { dispositivo }\end{array}$ & Elementos comuns & Raciocínio & Exemplos de referência loT \\
\hline $\begin{array}{l}\text { Configuração do } \\
\text { dispositivo: A } \\
\text { configuracão do } \\
\text { software do dispositivo } \\
\text { loT pode ser alterada } \\
\text { e tais alterações } \\
\text { podem ser realizadas } \\
\text { apenas por entidades } \\
\text { autorizadas. }\end{array}$ & $\begin{array}{l}\text { 1. A capacidade de alterar as } \\
\text { configurações de software do } \\
\text { dispositivo } \\
\text { 2. A capacidade de restringir alterações } \\
\text { de configuração apenas a entidades } \\
\text { autorizadas } \\
\text { 3. A capacidade das entidades } \\
\text { autorizadas de restaurar o dispositivo } \\
\text { para uma configuração segura definida } \\
\text { por uma entidade autorizada }\end{array}$ & $\begin{array}{l}\text { - Esse recurso oferece suporte ao gerenciamento de } \\
\text { vulnerabilidades, de acesso, proteção de dados e } \\
\text { detecção de incidentes. } \\
\text { - Uma entidade autorizada pode querer alterar a } \\
\text { configuração de um dispositivo por várias razões, } \\
\text { incluindo a segurança cibernética, } \\
\text { interoperabilidade, privacidade e usabilidade. Sem } \\
\text { um recurso de configuração de dispositivo, uma } \\
\text { entidade autorizada não pode customizar um } \\
\text { dispositivo para atender às suas necessidades, ou } \\
\text { integrar o dispositivo ao ambiente da entidade } \\
\text { autorizada etc. } \\
\text { - A maioria dos recursos de segurança cibernética } \\
\text { dependem, em certo grau, da presença de um } \\
\text { recurso de configuração do dispositivo. } \\
\text { - Entidades não autorizadas podem querer alterar a } \\
\text { configuração de um dispositivo por vários motivos, } \\
\text { como obter acesso não autorizado, causar o mau } \\
\text { funcionamento do dispositivo ou monitorar } \\
\text { secretamente o ambiente do dispositivo. } \\
\text { - A capacidade de restaurar uma configuração } \\
\text { segura para um dispositivo é bastante útil quando a } \\
\text { configuração atual contém erros, foi danificada ou } \\
\text { corrompida, ou não é mais considerada confiável. }\end{array}$ & $\begin{array}{l}\text { - BITAG: } 7.1 \\
\text { - CSA: } 22 \\
\text { - ENISA: GP-TM-06 } \\
\text { - IEC: CR 7.4, CR } 7.6 \\
\text { - IIC: } 7.3,7.6,8.10,11.5 \\
\text { - IoTSF: } 2.4 .8 .17,2.4 .15 \\
\text { - ISOC/OTA: } 26 \\
\text { - OCF: } 5.3 .3,8.2,12,13.3 .1 \\
\text { - PSA: C2.3, R6.1, R7.1 }\end{array}$ \\
\hline
\end{tabular}




\begin{tabular}{|c|c|c|c|}
\hline $\begin{array}{c}\text { Recurso de } \\
\text { cibersegurança do } \\
\text { dispositivo }\end{array}$ & Elementos comuns & Raciocínio & Exemplos de referência loT \\
\hline $\begin{array}{l}\text { Proteção de dados: } \\
\text { O dispositivo loT pode } \\
\text { proteger os dados que } \\
\text { armazena e transmite } \\
\text { contra acesso e } \\
\text { modificação não } \\
\text { autorizados. }\end{array}$ & $\begin{array}{l}\text { 1. A capacidade de usar módulos } \\
\text { criptográficos comprovadamente } \\
\text { seguros para algoritmos criptográficos } \\
\text { padronizados (por exemplo, } \\
\text { criptografia com autenticação, hashes } \\
\text { criptográficos, validação de assinatura } \\
\text { digital) para evitar que a } \\
\text { confidencialidade e integridade dos } \\
\text { dados armazenados e transmitidos do } \\
\text { dispositivo sejam comprometidos } \\
\text { 2. A capacidade das entidades } \\
\text { autorizadas de tornar todos os dados } \\
\text { no dispositivo inacessíveis por todas } \\
\text { as entidades, previamente autorizadas } \\
\text { ou não (por exemplo, por meio de } \\
\text { limpeza do armazenamento interno, } \\
\text { destruição de chaves criptográficas } \\
\text { para dados criptografados) } \\
\text { 3efinições de configuração para uso } \\
\text { com o recurso de configuração do } \\
\text { dispositivo, incluindo, mas não se } \\
\text { limitando a, capacidade de entidades } \\
\text { autorizadas de configurar o uso } \\
\text { próprio de criptografia, como escolher } \\
\text { um comprimento de chave }\end{array}$ & $\begin{array}{l}\text { - Esse recurso oferece suporte ao gerenciamento de } \\
\text { acesso, proteção de dados e detecção de } \\
\text { incidentes. } \\
\text { - Entidades autorizadas (por exemplo, clientes, } \\
\text { administradores, usuários) geralmente desejam que } \\
\text { a confidencialidade de seus dados seja protegida } \\
\text { para que entidades não autorizadas não possam } \\
\text { acessar os dados e usá-los indevidamente. } \\
\text { - As entidades autorizadas muitas vezes desejam } \\
\text { que a integridade de seus dados seja protegida } \\
\text { para que não sejam inadvertidamente ou } \\
\text { intencionalmente alterados, o que pode provocar } \\
\text { várias consequências adversas (por exemplo, emitir } \\
\text { o comando errado para uma peça de equipamento, } \\
\text { ocultando atividades maliciosas). }\end{array}$ & $\begin{array}{l}\text { - AGELIGHT: } 5,7,18,24,25 \text {, } \\
34 \\
\text { - BITAG: } 7.2,7.10 \\
\text { - CSDE: } 5.1 .3,5.1 .4,5.1 .5, \\
\text { 5.1.8, 5.1.10 } \\
\text { - CTIA: } 4.8,5.14,5.15 \\
\text { - ENISA: GP-OP-04, GP-TM- } \\
\text { O2, GP-TM-04, GP-TM-14, } \\
\text { GP-TM-24, GP-TM-32, GP- } \\
\text { TM-34, GP-TM-35, GP-TM- } \\
\text { 39, GP-TM-40 } \\
\text { - ETSI: } 4.4-1,4.5-1,4.5-2, \\
\text { 4.11-1, 4.11-2, 4.11-3 } \\
\text { - GSMA: CLP136.4.1.1, 6.11, } \\
\text { 6.12.1.1, 6.19, 7.6.1, } \\
\text { 8.10.1.1, 8.11.1 } \\
\text { - IEC: CR 3.1, CR 3.4, CR 4.1, } \\
\text { CR 4.2, CR 4.3 } \\
\text { - IIC: } 7.3,7.4,7.6,7.7,8.8, \\
\text { 8.11, 8.13, 9.1, 10.4, 11.9 } \\
\text { - IoTSF: 2.4.6.5, 2.4.7, 2.4.8.8, } \\
\text { 2.4.8.16, 2.4.9, 2.4.12.2, } \\
\text { 2.4.16.1, 2.4.16.2 } \\
\text { - ISOC/OTA: 2, 17, 33 } \\
\text { - OCF: 8.2, 11.2.1, 11.3, } \\
\text { 14.2.2 } \\
\text { - PSA: C1.1, C1.4, C2.4, D5.2, } \\
\text { R2.2, R2.3, R6.1, R7.1 }\end{array}$ \\
\hline
\end{tabular}




\begin{tabular}{|c|c|c|c|}
\hline $\begin{array}{c}\text { Recurso de } \\
\text { cibersegurança do } \\
\text { dispositivo } \\
\end{array}$ & Elementos comuns & Raciocínio & Exemplos de referência loT \\
\hline $\begin{array}{l}\text { Acesso lógico às } \\
\text { interfaces: } O \\
\text { dispositivo loT pode } \\
\text { restringir o acesso } \\
\text { lógico às suas } \\
\text { interfaces locais e de } \\
\text { rede, e aos protocolos } \\
\text { e serviços usados por } \\
\text { essas interfaces, } \\
\text { apenas para entidades } \\
\text { autorizadas. }\end{array}$ & $\begin{array}{l}\text { 1. A capacidade de desativar lógica ou } \\
\text { fisicamente quaisquer interfaces locais } \\
\text { e de rede que não sejam necessárias } \\
\text { para a funcionalidade do núcleo do } \\
\text { dispositivo } \\
\text { 2. A capacidade de restringir logicamente } \\
\text { o acesso a cada interface de rede } \\
\text { apenas a entidades autorizadas (por } \\
\text { exemplo, autenticação de dispositivo, } \\
\text { autenticação de usuário) } \\
\text { 3. Definições de configuração para uso } \\
\text { com o recurso de configuração do } \\
\text { dispositivo, incluindo, dentre outros, } \\
\text { a capacidade de ativar, desativar e } \\
\text { ajustar os limites para qualquer } \\
\text { capacidade que o dispositivo possa ter } \\
\text { para bloquear ou desativar uma conta, } \\
\text { ou para atrasar tentativas de } \\
\text { autenticação adicionais após muitas } \\
\text { tentativas de autenticação fracassadas }\end{array}$ & $\begin{array}{l}\text { - Esse recurso oferece suporte ao gerenciamento de } \\
\text { vulnerabilidades, de acesso, proteção de dados e } \\
\text { detecção de incidentes. } \\
\text { - Limitar o acesso a interfaces reduz a superfície de } \\
\text { ataque do dispositivo, dando aos invasores menos } \\
\text { oportunidades de comprometê-lo. Por exemplo, o } \\
\text { acesso irrestrito à rede por um dispositivo loT } \\
\text { permite que os invasores interajam diretamente } \\
\text { com o dispositivo, o que aumenta significativamente } \\
\text { a probabilidade de o dispositivo ficar comprometido. } \\
\text { - O acesso às interfaces pode ser parcial ou } \\
\text { totalmente limitado com base no estado do } \\
\text { dispositivo. Por exemplo, se um dispositivo não tiver } \\
\text { sido provisionado com credenciais de rede } \\
\text { adequadas, todo o acesso de/para interfaces de } \\
\text { rede será limitado se um esquema de integração } \\
\text { seguro for usado. }\end{array}$ & $\begin{array}{l}\text { - AGELIGHT: } 10,13,14,15, \\
\text { 16, } 19 \\
\text { - BITAG: } 7.1,7.2,7.3,7.6 \\
\text { - CSA: } 2,4,20 \\
\text { - CSDE: } 5.1 .2 \\
\text { - CTIA: } 3.2,3.3,3.4,4.2,4.3, \\
\text { 4.9, } 5.2 \\
\text { - ENISA: GP-TM-08, GP-TM- } \\
\text { 09, GP-TM-21, GP-TM-22, } \\
\text { GP-TM-25, GP-TM-27, GP- } \\
\text { TM-29, GP-TM-33, GP-TM- } \\
\text { 42, GP-TM-44, GP-TM-45 } \\
\text { - ETSI: } 4.1-1,4.4-1,4.6-1,4.6- \\
\text { 2 } \\
\text { - GSMA: CLP13-6.9.1, 6.12.1, } \\
\text { 6.20.1, 7.6.1, 8.2.1, 8.4.1 } \\
\text { - IEC: CR 1.1, CR 1.2, CR 1.5, } \\
\text { CR 1.7, CR 1.11, CR 2.1, CR } \\
\text { 2.2, CR 2.13, CR 7.7, EDR } \\
\text { 2.13 } \\
\text { - IIC: } 7.3,7.4,8.3,8.6,11.7 \\
\text { - IoTSF: } 2.4 .4 .5,2.4 .4 .9, \\
\text { 2.4.5.5, 2.4.6.3, 2.4.6.4, } \\
\text { 2.4.7, 2.4.8 } \\
\text { - ISOC/OTA: 3, 12, 13, 14, 15, } \\
\text { 16 } \\
\text { - NEMA: Redes de } \\
\text { segmentação, gerenciamento } \\
\text { de usuários, endurecimento } \\
\text { de dispositivos } \\
\text { - OCF: } 5.1,5.2,10,12 \\
\text { - PSA: C2.3, D2.1, D2.2, D2.3, } \\
\text { D2.4, D3.1 D3.3, R3.1, R3.2, } \\
\text { R3.3, R4.2, R4.5 R6.1 }\end{array}$ \\
\hline
\end{tabular}




\begin{tabular}{|c|c|c|c|}
\hline $\begin{array}{l}\text { Recurso de } \\
\text { cibersegurança do } \\
\text { dispositivo }\end{array}$ & Elementos comuns & Raciocínio & Exemplos de referência loT \\
\hline $\begin{array}{l}\text { Atualização do } \\
\text { Software: O software } \\
\text { do dispositivo loT } \\
\text { pode ser atualizado } \\
\text { por entidades } \\
\text { autorizadas apenas } \\
\text { usando um } \\
\text { mecanismo seguro e } \\
\text { configurável. }\end{array}$ & $\begin{array}{l}\text { 1. A capacidade de atualizar o software } \\
\text { do dispositivo por meio remoto (por } \\
\text { exemplo, download de rede) e/ou } \\
\text { meios locais (ex: mídia removível) } \\
\text { 2. A capacidade de verificar e autenticar } \\
\text { qualquer atualização antes de ser } \\
\text { instalada } \\
\text { 3. A capacidade das entidades } \\
\text { autorizadas reverterem o software } \\
\text { atualizado para uma versão anterior } \\
\text { 4. A capacidade de restringir ações de } \\
\text { atualização apenas a entidades } \\
\text { autorizadas } \\
\text { 5. A capacidade de ativar ou desativar a } \\
\text { atualização } \\
\text { 6. Definições de configuração para uso } \\
\text { com o recurso de configuração do } \\
\text { dispositivo, incluindo, por exemplo: } \\
\text { a. A capacidade de configurar } \\
\text { qualquer mecanismo de } \\
\text { atualização remota para ser } \\
\text { iniciado automática ou } \\
\text { manualmente para downloads e } \\
\text { instalações de atualização } \\
\text { b. A capacidade de ativar ou } \\
\text { desativar a notificação quando } \\
\text { uma atualização está disponível e } \\
\text { especificar quem ou o que deve } \\
\text { ser notificado }\end{array}$ & $\begin{array}{l}\text { - Esse recurso oferece suporte ao gerenciamento de } \\
\text { vulnerabilidades. } \\
\text { - As atualizações podem remover vulnerabilidades de } \\
\text { um dispositivo loT, o que diminui a probabilidade de } \\
\text { um invasor comprometer o dispositivo. } \\
\text { - As atualizações podem corrigir problemas } \\
\text { operacionais do dispositivo loT, o que pode } \\
\text { melhorar a disponibilidade, confiabilidade, } \\
\text { desempenho e outros aspectos da operação do } \\
\text { dispositivo. } \\
\text { - Algumas entidades autorizadas precisarão de } \\
\text { recursos de atualização automática para atender a } \\
\text { seus objetivos e necessidades de segurança } \\
\text { cibernética, enquanto outras preferem ou precisam } \\
\text { de um controle mais direto sobre as atualizações e } \\
\text { seus aplicativos. } \\
\text { - Algumas organizações podem querer um recurso } \\
\text { de reversão no caso de uma atualização afetar } \\
\text { inadvertidamente aplicativos críticos ou integração } \\
\text { com outros sistemas, enquanto outras organizações } \\
\text { podem preferir eliminar o risco de alguém } \\
\text { intencional ou inadvertidamente reverter o software } \\
\text { para uma versão vulnerável. }\end{array}$ & $\begin{array}{l}\text { - AGELIGHT: } 1,2,4 \\
\text { - } \text { BITAG: } 7.1 \\
\text { - CSDE: } 5.1 .9 \\
\text { - CTIA: } 3.5,3.6,4.5,4.6,5.5 \text {, } \\
\text { 5.6 } \\
\text { - ENISA: GP-TM-05, GP-TM- } \\
\text { 06, GP-TM-18, GP-TM-19 } \\
\text { - ETSI: } 4.3-1,4.3-2,4.3-7 \\
\text { - GSMA: } 7.5 .1 \\
\text { - IEC: CR 3.4, EDR 3.10 } \\
\text { - IIC: } 7.3,11.5 .1 \\
\text { - IoTSF: } 2.4 .5 .1,2.4 .5 .2, \\
2.4 .5 .3,2.4 .5 .4,2.4 .5 .8, \\
2.4 .6 .1 \\
\text { - ISOC/OTA: 1, 6, } 8 \\
\text { - NEMA: Updating Devices } \\
\text { - OCF: } 14.5 \\
\text { - PSA: C2.1, C2.2, R1.1, R1.2, } \\
\text { R6.1 }\end{array}$ \\
\hline
\end{tabular}




\begin{tabular}{|c|c|c|c|}
\hline $\begin{array}{l}\text { Recurso de } \\
\text { cibersegurança do } \\
\text { dispositivo }\end{array}$ & Elementos comuns & Raciocínio & Exemplos de referência loT \\
\hline $\begin{array}{l}\text { Conscientização } \\
\text { sobre o estado de } \\
\text { cibersegurança: O } \\
\text { dispositivo loT pode } \\
\text { relatar seu estado de } \\
\text { seguranca cibernética } \\
\text { e tornar essas } \\
\text { informações } \\
\text { acessíveis apenas a } \\
\text { entidades autorizadas. }\end{array}$ & $\begin{array}{l}\text { 1. A capacidade de relatar o estado de } \\
\text { cibersegurança do dispositivo } \\
\text { 2. A capacidade de diferenciar entre } \\
\text { quando um dispositivo provavelmente } \\
\text { operará conforme o esperado e } \\
\text { quando pode estar em um estado de } \\
\text { segurança cibernética degradado } \\
\text { 3. A capacidade de restringir o acesso ao } \\
\text { indicador de estado para que apenas } \\
\text { entidades autorizadas possam } \\
\text { visualizá-lo } \\
\text { 4. A capacidade de evitar que quaisquer } \\
\text { entidades (autorizadas ou não } \\
\text { autorizadas) editem o estado, exceto } \\
\text { aquelas entidades que são } \\
\text { responsáveis por manter as } \\
\text { informações de estado do dispositivo } \\
\text { 5. A capacidade de disponibilizar as } \\
\text { informações de estado para um } \\
\text { serviço em outro dispositivo, como um } \\
\text { servidor de registro de } \\
\text { eventos/estados }\end{array}$ & $\begin{array}{l}\text { - Esse recurso oferece suporte ao gerenciamento de } \\
\text { vulnerabilidade e detecção de incidentes. } \\
\text { - A conscientização do estado da cibersegurança } \\
\text { ajuda a permitir a investigação de } \\
\text { comprometimentos, a identificação do uso indevido, } \\
\text { e a solução de certos problemas operacionais. } \\
\text { - Como o dispositivo torna outras entidades cientes } \\
\text { de um estado de segurança cibernética irá variar } \\
\text { com base nas necessidades e objetivos específicos } \\
\text { do contexto, mas pode incluir a captura e registro } \\
\text { de informações sobre eventos em um registro } \\
\text { persistente que pode ter que ser armazenado fora } \\
\text { do dispositivo, enviando sinais para um sistema de } \\
\text { monitoramento a ser tratado externamente ou } \\
\text { alertando por meio de uma interface no próprio } \\
\text { dispositivo loT. }\end{array}$ & $\begin{array}{l}\text { - CSDE: } 5.1 .7 \\
\text { - CTIA: } 4.7,4.12,5.7,5.16 \\
\text { - ENISA: GP-TM-55, GP-TM- } \\
56 \\
\text { - ETSI: 4.7-2, 4.10-1 } \\
\text { - GSMA: CLP13_6.13.1, 7.2.1, } \\
\text { 9.1.1.2 } \\
\text { - IEC: CR 2.8, CR 3.9, CR 6.1, } \\
\text { CR } 6.2 \\
\text { - IIC: } 7.3,7.5,7.7,8.9,10.3 \text {, } \\
\text { 10.4 } \\
\text { - IoTSF: 2.4.7.5 } \\
\text { - NEMA: Monitoring Devices } \\
\text { and Systems [Dispositivos e } \\
\text { Sistemas de Monitoramento] } \\
\text { - OCF: } 5.1,5.7,8.6,12,13.8, \\
\text { 13.16 } \\
\text { PSA: C1.3, D1.1, D3.2, D3.4, } \\
\text { D3.5, D5.1, R4.1, R4.3, R4.4 }\end{array}$ \\
\hline
\end{tabular}


Referências

[1] Iniciativa de Transformação da Força Tarefa Conjunta (2013) Segurança e Controles de Privacidade para Organizações e Sistemas de Informação Federais. (Instituto Nacional de Normas e Tecnologia, Gaithersburg, MD), Publicação Especial do NIST (SP) 80053, Rev. 4, Inclui atualizações a partir de 22 de janeiro de 2015. https://doi.org/10.6028/NIST.SP.800-53r4

[2] Fagan M, Megas KN, Scarfone K, Smith M (2020) Atividades básicas de segurança cibernética para fabricantes de dispositivos IoT. (Instituto Nacional de Normas e Tecnologia, Gaithersburg, MD), Relatório Interno ou Interagências do NIST (IR) 8259. https://doi.org/10.6028/NIST.IR.8259

[3] Iniciativa de Transformação da Força Tarefa Conjunta (2012) Guia para a Realização de Avaliações de Risco. (Instituto Nacional de Normas e Tecnologia, Gaithersburg, MD), Publicação Especial do NIST (SP) 800-30 Rev. 4. https://doi.org/10.6028/NIST.SP.800-30r1

[4] Boeckl K, Fagan M, Fisher W, Lefkovitz N, Megas K, Nadeau E, Piccarreta B, Gabel O'Rourke D, Scarfone K (2019) Considerações para gerenciar riscos de segurança cibernética e privacidade da Internet das Coisas (IoT). (Instituto Nacional de Normas e Tecnologia, Gaithersburg, MD), NIST Interagência ou Relatório Interno (IR) 8228. https://doi.org/10.6028/NIST.IR.8228

[5] AgeLight Digital Trust Advisory Group (2019) Arquitetura de Segurança IoT e Caixa de Ferramentas de Risco (IoTSA) v3.1. (AgeLight Advisory \& Research Group, Bellevue, WA). http://agelight.com/iot.html

[6] Broadband Internet Technical Advisory Group (BITAG) (2016) Recomendações de Segurança e Privacidade da Internet das Coisas (IoT). (Grupo de Consultoria Técnica de Banda Larga da Internet [BITAG], Denver, CO). https://www.bitag.org/documents/BITAG_Report_Internet_of_Things_(IoT)_Security_and_Privacy_Recommendations.pdf

[7] Cloud Security Alliance (CSA) IoT Working Group (2015) Identity and Access Management for the Internet of Things [Grupo de trabalho da IoT (2015) Gerenciamento de identidade e acesso para a Internet das Coisas]. (Cloud Security Alliance [CSA]). https://cloudsecurityalliance.org/download/identity-and-accessmanagement-for-the-iot/

[8] Council to Secure the Digital Economy (CSDE), "The C2 Consensus on IoT Device Security Baseline Capabilities" (Consenso do C2 sobre Recursos de linha de base de segurança do dispositivo IoT). (Conselho de Segurança da Economia Digital [CSDE]). https://securingdigitaleconomy.org/wp-content/uploads/2019/09/CSDE_IoT-C2Consensus-Report_FINAL.pdf

[9] CTIA (2018), "CTIA Cybersecurity Certification Test Plan for IoT Devices, Version 1.0.1 (Teste de certificação de cibersegurança para dispositivos IoT) " (CTIA, Washington, DC). https://www.ctia.org/about-ctia/test-plans/ 
[10] Agência da União Europeia para a Segurança das Redes e da Informação (ENISA) (2017) , "Recomendações de Segurança da linha de base para IoT no Contexto de Infraestruturas Críticas de Informação" Agência da União Europeia para a Segurança de Rede e da Informação [ENISA], Atenas, Grécia) https://www.enisa.europa.eu/publications/baseline-security-recommendations-for-iot

[11] Instituto Europeu de Padrões em Telecomunicações (ETSI), "Segurança cibernética para o consumidor da Internet das Coisas" Especificações Técnicas do ETSI 103645 V1.1.1. ${ }^{2}$ Instituto Europeu de Padrões em Telecomunicações [ETSI], Sophia Antipolis Cedex, França).

https://www.etsi.org/deliver/etsi_ts/103600_103699/103645/01.01.01_60/ts_103645v01 0101p.pdf

[12] Groupe Spéciale Mobile Association (GSMA), “Avaliação de Segurança IoT da GSMA" (Groupe Spéciale Mobile Association [GSMA], Londres, Reino Unido). https://www.gsma.com/iot/iot-security-assessment/

[13] Comissão Eletrotécnica Internacional (IEC) (2019), "IEC 62443-4-2, Edição 1.0, Segurança para automação industrial e sistemas de controle - Parte 4-2: Requisitos de segurança técnica para componentes IACS (Comissão Eletrotécnica Internacional) [IEC], Genebra, Suíça). https://webstore.iec.ch/publication/34421

[14] Consórcio da Internet Industrial (IIC), Internet Industrial das Coisas Volume G4: Security Framework" (Consórcio da Internet Industrial [IIC], Needham, MA). https://www.iiconsortium.org/IISF.htm

[15] Base de Segurança IoT (IoTSF) (2018), "Estrutura de Compliance em Segurança de IoT, Lançamento 2" (Base de Segurança IoT [IoTSF], Livingston, Escócia). https://www.iotsecurityfoundation.org/best-practice-guidelines/

[16] Aliança de Confiança Online (OTA) 2017), Estrutura de Privacidade e Segurança de IoT v2.5. (Online Trust Alliance [OTA], Uma Iniciativa da Sociedade da Internet). https://www.internetsociety.org/resources/doc/2018/iot-trust-framework-v2-5/

[17] Associação Nacional de Fabricantes Elétricos (NEMA) (2018), "Melhores Práticas de Higiene Cibernética" (Associação Nacional de Fabricantes Elétricos [NEMA], Rosslyn, VA). https://www.nema.org/Standards/Pages/Cyber-Hygiene-Best-Practices.aspx

[18] Fundação de Conectividade Aberta (OCF)(2020) Especificações de Segurança da OCF Versão 2.1.2 (Fundação de Conectividade Aberta [OCF], Beaverton, Oregon). https://openconnectivity.org/specs/OCF_Security_Specification_v2.1.2.pdf

[19] Platform Security Architecture (PSA) Arquitetura de Segurança de Plataforma Membros do Acordo Conjunto das Partes Interessadas (JSA) (2020), "Certificação PSA"TM Questionário de Nível I Versão 2.0 Beta” (Arm Limited, Cambridge, Reino Unido). https://www.psacertified.org/security-certification/psa-certified-level-1

[20] Johnson A, Dempsey K, Ross R, Gupta S, Bailey D (2011) Guia para gerenciamento de configurações com foco na segurança de sistemas de informação. (Instituto Nacional de Padrões e Tecnologia, Gaithersburg, MD), Publicação Especial do NIST (SP) 800-128. https://doi.org/10.6028/NIST.SP.800-128

2 O ETSI está atualmente desenvolvendo o Padrão Europeu ETSI 303 645, que é semelhante, mas não idêntico à Especificação Técnica 103645 citada aqui. A versão 303645 não é usada nesta publicação porque ainda é um esboço. 
[21] Barker E, Chen L, Roginsky A, Vassilev A, Davis R (2019) Recomendação para esquemas de estabelecimento de chaves de codificação usando criptografia de logaritmo discreto (Instituto Nacional de Normas e Tecnologia, Gaithersburg, MD), Publicação Especial do NIST (SP) 800-56A, Rev. 3. https://doi.org/10.6028/NIST.SP.800-56Ar3

[22] Comitê Nacional de Sistemas de Segurança (2015) Glossário do Comitê Nacional de Sistemas de Segurança (CNSS). (Agência de Segurança Nacional, Ft. Meade, MD), Instrução CNSS (CNSSI) No. 4009.

[23] Souppaya M, Scarfone K (2013) Guia para tecnologias de gerenciamento de patches corporativos. (Instituto Nacional de Normas e Tecnologia, Gaithersburg, MD), Publicação Especial do NIST (SP) 800-40 Rev. 3. https://doi.org/10.6028/NIST.SP.800-40r3 


\section{Appendix A-Compreendendo a linha de base do núcleo para recursos de cibersegurança de dispositivo loT no contexto das necessidades e objetivos do cliente}

As organizações devem estar cientes de que os recursos apresentados na Table 1 são um ponto de partida para ajudar a fornecer os meios pelos quais as partes interessadas podem atender às necessidades e objetivos comuns de segurança cibernética. As áreas de mitigação de risco que os clientes necessitam incentivam a busca por uma maneira de identificar as necessidades e metas de segurança cibernética proporcionadas por um dispositivo IoT, utilizando-se os recursos de segurança cibernética do dispositivo. Por exemplo, Figure 1 mostra as áreas de mitigação de risco e os desafios definidos no NISTIR 8228, Considerações para Gerenciar Riscos de Privacidade e Segurança Cibernética na Internet das Coisas (IoT) [3] que podem receber suporte parcial através dos recursos definidos na Table 1. O NISTIR 8228 cita desafios adicionais que os recursos de segurança cibernética do dispositivo principal não abordam porque esses desafios geralmente se aplicam a relativamente poucos dispositivos IoT em comparação com a aplicabilidade dos recursos principais.

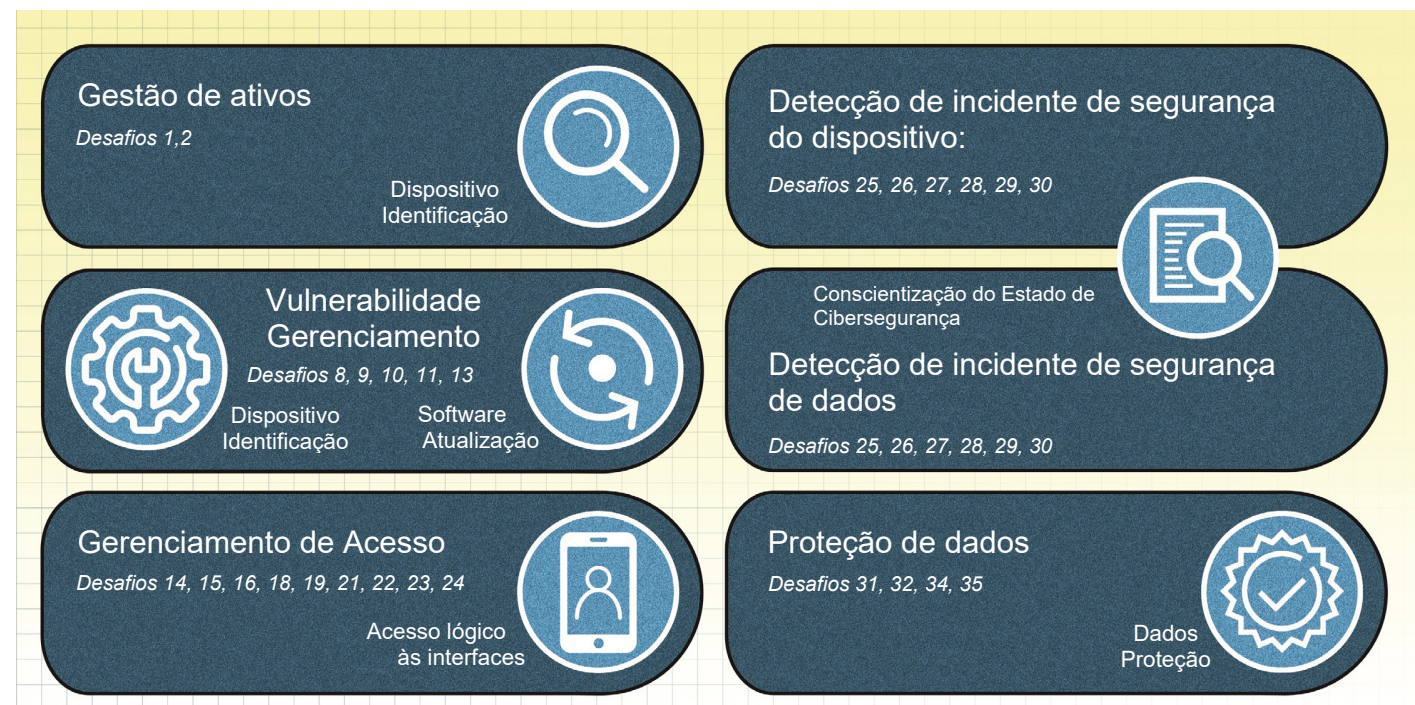

Figura 1: NISTIR 8228 Áreas de mitigação de risco suportadas por cada recurso de cibersegurança do núcleo do dispositivo

Figure 1 Demonstra que um conjunto amplo e comum de áreas de mitigação de risco foi considerado para a linha de base do núcleo, que deve ser compreendido pelos fabricantes e outros leitores como ponto inicial. Embora os dispositivos IoT que fornecem os recursos básicos de cibersegurança descritos na linha de base do núcleo possam ajudar os clientes a suprir mais facilmente as suas necessidades e objetivos de cibersegurança, na realidade, os clientes provavelmente se concentrarão em áreas de mitigação de risco diferentes e mais específicas. Portanto, os seis recursos e elementos comuns na Table 1 não devem ser considerados como sendo uma definição universal e na íntegra dos recursos de cibersegurança de dispositivo necessários para todos os clientes.

Conforme descrito no NISTIR 8259, os fabricantes devem considerar os clientes potenciais e casos de uso para seus dispositivos IoT, para identificar precisamente quais os recursos de cibersegurança serão necessários, dependendo do contexto. Os fabricantes podem definir ainda 
mais quais os recursos de cibersegurança devem acrescentar, com base no conhecimento e na pesquisa sobre os seus clientes. Isso pode exigir a incorporação de novos recursos para oferecer suporte a mitigações de risco em outras áreas além do que está sendo exposto aqui (ex: teste de penetração ou outras formas de teste/validação de componentes, arquiteturas de rede específicas para reduzir o risco) ou incorporar elementos mais exclusivos para um dispositivo IoT para que ele ofereça suporte de forma menos ampla, porém, necessário no contexto de determinado cliente ou caso de uso, mitigações de risco críticas, e outras necessidades e objetivos de cibersegurança. Os fabricantes devem imaginar possibilidades além das mitigações de risco que podem afetar a segurança cibernética do dispositivo e seus elementos, ou seja, a usabilidade com base no cliente e casos de uso, funções e responsabilidades relacionadas à cibersegurança, e como os clientes esperam que os dispositivos sejam distribuídos. Outros aspectos importantes de risco são as necessidades e objetivos de segurança cibernética social (ex: proteção contra o desenvolvimento de botnets) que podem não estar diretamente refletidos nas necessidades e objetivos do cliente, mas devem ser considerados. 


\section{Appendix B-Glossário}

Os termos selecionados e utilizados neste documento são definidos abaixo.

Entidade autorizada

Configuração

Linha de base do núcleo

Estado de Cibersegurança

Estado degradado de cibersegurança

Linha de base do núcleo para recursos de cibersegurança do dispositivo

Identificador de dispositivo

\section{Entidade}

Uma entidade que, implícita ou explicitamente, recebeu aprovação para interagir com um dispositivo IoT específico. Os recursos de cibersegurança do dispositivo na linha de base principal não especificam como a autorização é implementada para distinguir entidades autorizadas e não autorizadas, mas podem incluir gerenciamento de identidade e autenticação para estabelecer a autorização de entidades. Cabe à organização decidir como cada dispositivo implementará a autorização. Além disso, uma entidade autorizada a interagir com um dispositivo IoT de uma maneira pode não estar autorizada a interagir com o mesmo dispositivo de outra maneira.

"As possíveis condições, parâmetros e especificações com os quais um sistema de informação ou componente do sistema pode ser descrito ou organizado [20]." O recurso de configuração do dispositivo não define quais configurações devem existir, apenas estabelece que existe um mecanismo para gerenciar as configurações.

Um conjunto de recursos técnicos de dispositivos necessários para dar suporte a controles comuns de segurança cibernética que protegem os dispositivos do cliente e seus dados, sistemas e ecossistemas.

A condição de cibersegurança que um dispositivo expressa de forma significativa e útil para entidades autorizadas. Por exemplo, um dispositivo muito simples pode expressar seu estado em termos de estar ou não operando conforme o esperado, enquanto um dispositivo complexo pode realizar o registro de segurança cibernética, verificar sua integridade na inicialização e relatar os resultados, e examinar e relatar aspectos adicionais sobre o seu estado de cibersegurança.

Um estado de cibersegurança que indica que a cibersegurança do dispositivo foi significativamente afetada de forma negativa, como um dispositivo incapaz de operar conforme esperado, ou a integridade do software do dispositivo foi violada.

Veja linha de base do núcleo.

Um valor exclusivo de contexto - um valor exclusivo dentro de um contexto específico - que está associado a um dispositivo (ex: um string que consiste de um endereço de rede). (Esta definição é derivada da [21].)

Uma pessoa, dispositivo, serviço, rede, domínio, fabricante ou outra parte que possa interagir com um dispositivo IoT. 
Interfaces Um limite entre o dispositivo IoT e as entidades onde ocorrem as interações. (Esta definição é derivada de [22].) Existem dois tipos de interfaces: rede e local.

Interface Local Uma interface que só pode ser acessada fisicamente, como uma porta (por exemplo, USB, áudio, vídeo / display, serial, paralelo, Thunderbolt) ou uma unidade de mídia removível (por exemplo, unidade de CD / DVD, slot de cartão de memória).

Identificador Um identificador de dispositivo que é expresso logicamente pelo software do Lógico dispositivo. Um exemplo é um endereço de controle de acesso à mídia (MAC) atribuído a uma interface de rede.

Interface de Uma interface que conecta o dispositivo IoT a uma rede.

rede

Identificador Um identificador de dispositivo que é expresso fisicamente pelo dispositivo

Físico (por exemplo, impresso na caixa de um dispositivo, exibido na tela de um dispositivo).

Software "Programas de computador e dados associados que podem ser escritos ou modificados dinamicamente durante a execução do dispositivo" (por exemplo, código de aplicativo, bibliotecas) [1].

Atualização Um patch, atualização ou outra modificação no código que corrige problemas de segurança e/ou funcionalidade no software. (Esta definição é derivada do [23].) 